\title{
CIRUGÍA AMBULATORIA EN UN SERVICIO DE UROLOGÍA. ANÁLISIS DEL PERIODO 2003 - 2006
}

\author{
Pedro Carrión López, Manuel Cortiñas Sáenz', María José Fajardo', María José Donate \\ Moreno, Héctor Pastor Navarro, Benjamín Martínez Córcoles, Rafael Ruíz Mondéjar, Antonio \\ Salinas Sánchez, Julio Antonio Virseda Rodríguez y Llanos Martínez Gómez?.
}

Servicio de Urología. Anestesia y Reanimación?. Hospital de Nuestra Señora del Perpetuo Socorro. Complejo Hospitalario Universitario de Albacete. Albacete. España.

\begin{abstract}
Resumen.- OBJETIVO: En la cirugía sin ingreso deberemos distinguir entre el concepto de cirugía menor ambulatoria y cirugía mayor ambulatoria. La cirugía ambulatoria, permite que el paciente no esté en el hospital más allá de unas horas, mostrando una seguridad y efectividad similar a la cirugía convencional. Evaluar los resultados de un programa de cirugía ambulatoria.
\end{abstract}

MÉTODOS: Estudio descriptivo retrospectivo de los resultados de funcionamiento de la Cirugía Ambulatoria (C.A) en un Servicio de Urología, incluyendo 4185 pacientes en un periodo de estudio de cuatro años, que comprende desde el 1 de enero del 2003 hasta el 31 de diciembre del 2006.
RESULTADOS: En el periodo de estudio el índice de sustitución global fue del 83,6\%. El índice de ingresos fue del $2,5 \%$, de los cuales la mayoría fueron ingresos precoces. Las complicaciones mayores se presentaron en 26 pacientes $(0,6 \%)$, siendo la hemorragia mayor la predominante. La mayoría de las complicaciones son menores o leves, y el dolor en la zona de la herida quirúrgica es el problema encontrado con mayor frecuencia.

CONCLUSIONES: El auge y la promoción continua de la cirugía ambulatoria están más que justificadas. La alta satisfacción de los pacientes sometidos a este tipo de cirugía, con el escaso número de complicaciones registradas, nos proporcionan una valiosa herramienta de control del gasto sanitario.

Palabras clave: Cirugía ambulatoria. Servicio de Urología.

Summary.- OBJECTIVES: When talking about day surgery we have to differentiate between minor and major ambulatory surgery. Ambulatory surgery enables the patient to stay in the hospital not more than a few hours, showing similar safety and efficacy than conventional surgery. The objective of this paper is to evaluate the results of an ambulatory surgery program.

METHODS: Descriptive retrospective study of the results of the Ambulatory Surgery in a Urology Department, including 4185 patients in a four-year period, from January 7st 2003 to December 31 st 2006.

RESULTS: In the study period the global substitution rate was $83.6 \%$. The hospital admission rate was $2.5 \%$, most 
of which were early admissions. Major complications appeared in 26 patients $(0.6 \%)$, being major bleeding the predominant one. Most complications were minor or mild, and pain at the site of the surgical wound was the most frequent problem found.

CONCLUSIONS: The increase and continuous promotion of ambulatory surgery are more than justified. The high satisfaction among patients undergoing this type of surgery, with a very low number of complications registered, provides us with a valuable tool for health-care expenditure control.

Keywords: Ambulatory surgery. Urology department.

\section{INTRODUCCIÓN}

Ser intervenido quirúrgicamente, hasta hace pocos años, llevaba aparejado ineludiblemente un período más o menos largo de ingreso hospitalario. Actualmente, los procedimientos llamados de Cirugía Ambulatoria o de Alta Precoz (CA), permiten que el paciente no esté en el hospital más allá de unas horas. Esta modalidad quirúrgica ha demostrado similar seguridad y efectividad que la cirugía convencional o tradicional. De esta manera se obtiene un aumento del bienestar del paciente y su familia, con una disminución del coste por proceso al acortar la estancia hospitalaria, con unas complicaciones derivadas de esta actitud prácticamente despreciables $(1,2,3)$.

Dentro de la Cirugía sin ingreso se distinguen los conceptos de Cirugía Menor Ambulatoria y Cirugía Mayor Ambulatoria. La Cirugía Menor Ambulatoria incluye aquellas intervenciones que tienen como característica común la aplicación de procedimientos quirúrgicos sencillos y de corta duración, precisando en general de anestesia local, y en la que se procede al alta inmediata del paciente tras la realización de dicho acto (4). El término de Cirugía Mayor Ambulatoria (CMA) nace en 1986 con la publicación de un libro del mismo nombre publicado por Davis (5), apareciendo en España las primeras publicaciones de la mano de Vargas y cols. (2) en 1993. Es un modelo organizativo de asistencia quirúrgica basado en la seguridad y efectividad del tratamiento quirúrgico, el bienestar del paciente y eficiencia de la administración pública al no ser necesaria una cama de hospitalización dando el alta al paciente a las pocas horas de la intervención, independientemente del tipo de anestesia utilizada (local, troncular, locorregional o general) $(6,7)$.
En nuestro Hospital el programa de C.A comenzó en 1997, la actividad quirúrgica global ha sido de 76.310 intervenciones, de las cuales 42.142 han sido en régimen ambulatorio, repartidas entre las especialidades de Cirugía General, Cirugía Plástica, Traumatología, Ginecología, Oftalmología, Dermatología, Otorrinolaringología y Urología.

Con este trabajo pretendemos evaluar los resultados de un programa de cirugía ambulatoria y cuantificar las tasas de reingresos por complicaciones mayores y menores por años de estudio en pacientes intervenidos quirúrgicamente de diversas patologías urológicas en nuestro hospital.

\section{MATERIAL Y MÉTODOS}

Estudio descriptivo retrospectivo realizado en un hospital público de 252 camas, con un área sanitaria de 325.000 habitantes. El periodo de estudio comprende desde el 1 de enero del 2003 hasta el 31 de diciembre del 2006.

Los pacientes incluidos en el estudio fueron todos aquellos intervenidos por el Servicio de Urología en régimen de CMA. Se recogieron los diferentes tipos de anestesias realizadas a criterio del anestesiólogo y la comorbilidad asociada cuantificada por la escala de la American Society of Anesthesiologist (ASA). Se excluyeron del régimen quirúrgico de CMA los pacientes con estado físico basal altamente deteriorado por la comorbilidad asociada, aquellos catalogados por el Servicio de Anestesiología como ASA III no estables y ASA IV. En nuestro protocolo de CA. incluimos las siguientes intervenciones como cirugía menor ambulatoria: Vasectomía, fimosis, cistoscopias, escisión de lesiones locales y biopsias cerradas por aguja de próstata y como CMA: Varicocele, hidrocele, biopsia testicular, quistes epididiarios, cateterismos, criptorquidia, quistes de cordón, orquiectomía, prótesis testicular, meatoplastia, uretrotomía de corta duración, litotricia endoscópica, vasovasostomía, cirugía reparadora de pene y biopsia transuretral de vejiga. Los pacientes sometidos a cirugía menor ambulatoria fueron dados de alta de manera inmediata a la cirugía, mientras que en el caso de los pacientes sometidos a CMA, la primera fase del control postoperatorio se realizó en la Unidad de Recuperación Postanestésica (URPA), y posteriormente en las salas de readaptación al medio, y tras cumplir los criterios generales de alta se trasladaron a su domicilio. A las 24 horas del alta hospitalaria se realizó una llamada telefónica para conocer su evolución.

las variables a estudiar fueron: índice de sustitución, índice de suspensiones, tasa o índice de ingresos y complicaciones mayores y menores. 
El índice de sustitución es el porcentaje de intervenciones realizadas en régimen de $\mathrm{CA}$ respecto al total de intervenciones quirúrgicas urológicas del centro.

El índice de ingresos son los pacientes programados en régimen de $C A$ y que requieren ingreso hospitalario no planificado desde la URPA, definidos como ingresos precoces, o desde la Sala de Readaptación al medio, considerados como ingresos tardíos.

El índice de suspensiones corresponde a aquellas intervenciones no realizadas el día de la citación, bien sea por causas institucionales (error de programación, falta de instrumental, preparación incorrecta, falta de tiempo), causas atribuidas al paciente (rechazo intervención) y causas médicas (incumplimiento de criterios urológicos o anestésicos, enfermedad intercurrente).

El índice de reingresos, son aquellos pacientes que tras el alta hospitalaria, ingresan en el hospital por alguna complicación mayor o menor. Las complicaciones mayores se pueden definir como la respuesta adversa con potencial para provocar un daño grave. Se registraron la hemorragia mayor, angor prolongado, dolor torácico atípico, infarto agudo de miocardio, accidente cerebrovascular, broncoespasmo, bloqueo aurículo-ventricular, coma hiperglucémico, tromboflebitis, neumonía, shock anafiláctico, pancreatitis aguda, insuficiencia cardiaca descompensada, taquiarritmias. Las complicaciones menores se pueden definir como las respuestas adversas con mínimo potencial para producir un daño grave. Se registraron la hemorragia menor, dolor herida quirúrgica, cólico reno-ureteral, síncope de diversa etiología, dolor lumbar mecánico, retención urinaria, reacción alérgica, cefalea postpunción dural, vértigos, vómitos, fiebre, infección de herida quirúrgica, íleo paralítico, crisis de ansiedad, reagudización de EPOC, infección respiratoria e infección urinaria. Las complicaciones fueron recogidas a través del servicio de urgencias en el periodo de los 7 días posteriores al alta, y tratándose del único hospital público de nuestra zona sanitaria, el número de complicaciones mayores recogidas en el estudio se puede considerar real (no se han perdido datos), pero que el número de complicaciones menores ha podido sufrir sesgos al existir la posibilidad de que algunas hayan sido subsanadas en Atención Primaria y no hayamos tenido conciencia de su aparición. Se realiza un seguimiento durante un periodo de 30 días para la variable mortalidad. Se cuantificaron el número de episodios de infección de herida quirúrgica y el día de su aparición tras el alta hospitalaria.

\section{RESULTADOS}

Durante este periodo fueron intervenidos en régimen de CA 19.248 pacientes de manera global. Un $21,74 \%$ de las intervenciones (4.185 pacientes) correspondieron al Servicio de Urología. En dicho periodo, nuestro servicio atendió en cualquier modalidad quirúrgica a 5.002 pacientes, lo que implica un índice de sustitución global de $83,6 \%$. De todos ellos, fueron 274 mujeres (6,54\%) frente a 3.911 hombres $(93,45 \%)$. La edad media de los pacientes fue de 45 (DE: 21,34 ) años, con un rango entre 12 y 94 años.

Las intervenciones más realizadas fueron: 1.423 vasectomías, 1.325 circuncisiones, 242 excisión de lesiones locales, 222 biopsias cerradas por aguja de próstata, 204 hidrocelectomías, 193 cateterismos, 154 biopsias transuretrales de vejiga, 128 cistoscopias, 71 varicocelectomías, 51 biopsias abiertas de testículo, 38 quistectomías de epidídimo, 36 orquidopexias, 36 uretrotomías y meatoplastias, 24 orquiectomías, 22 cirugía reparadora de pene, 14 vasovasostomías, 2 prótesis testiculares. El tipo de anestesia seleccionada fue personalizada y basándose en criterios urológicos y anestésicos. La anestesia local, sola o combinada con sedación se empleo en 2.496 pacientes $(59,6 \%)$, la anestesia raquídea en 1.214 pacientes $(29 \%)$, mientras la anestesia general, únicamente en 353 pacientes $(8,4 \%)$, el resto de anestesias correspondieron a inducciones y epidurales (Figura 1).

El índice de ingresos fue de 104 pacientes $(2,5 \%)$, de los cuales, fueron ingresos precoces 87 pacientes $(2.1 \%)$, frente a los 17 pacientes $(0,4 \%)$ que representaron los ingresos tardíos.

\section{TIPOS DE ANESTESIA}

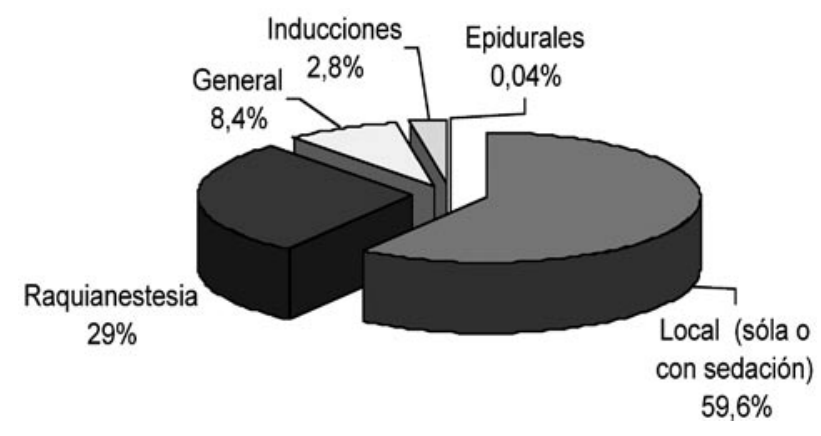

FIGURA 1. Porcentaje de uso de las diferentes técnicas anestésicas. 
El número de intervenciones no realizadas el día de la citación fue de 502 pacientes, con un índice de suspensión global del 12.2\%. La causa más frecuente fueron aquellas atribuidas a problemas médicos, principalmente la existencia de una enfermedad intercurrente. Las causas de suspensión atribuibles al paciente y a la institución representaron de forma conjunta un $17 \%$.

Las complicaciones mayores se presentaron en 26 pacientes $(0,6 \%)$, conformando un riesgo proporcional de aparición de 1:160 intervenciones. La complicación mayor más frecuente fue la hemorragia mayor en seis pacientes (23\%), presentándose en la serie episodios de taquiarritmias (3), neumonías (2), angor prolongados (2), insuficiencias cardiacas descompensadas (2) y tromboflebitis (2), como complicaciones mayores de importante morbilidad (Tabla I). Las complicaciones menores se presentaron en el $3,6 \%$ de las 4.185 intervenciones, con un riesgo proporcional de 1:28 intervenciones. De todas ellas, las más frecuentes fueron el dolor de la herida quirúrgica en 41 pacientes $(27 \%), 35$ pacientes presentaron hemorragia menor $(23 \%), 18$ infecciones de herida quirúrgica $(11,9 \%), 10$ manifestaron clínica de infección urinaria $(6,6 \%), 8$ cólicos nefríticos $(5,2 \%)$, y el resto de complicaciones menores, citadas con anterioridad, presen- taron una incidencia menor y se presentan en la Tabla II. La mortalidad a los 30 días de la cohorte fue de un 0,047\% (2 pacientes). El 100\% de los episodios de infección de herida quirúrgica aparecen en el día 16 tras la intervención en régimen de CA (Figura 2).

En la encuesta telefónica a las 24 horas de la intervención, el 96,8\% de los encuestados respondieron que se intervendrían de nuevo en régimen de $C$.A, y el $70,5 \%$ afirmaron que le evitamos importantes trastornos familiares con esta modalidad quirúrgica.

\section{DISCUSIÓN}

La cirugía sin ingreso pretende minimizar las complicaciones y que el paciente regrese a su entorno lo antes posible sin alterar la efectividad del acto quirúrgico, para lo cual han tenido que imponerse técnicas con mínimos efectos secundarios y rápida recuperación, donde los pacientes y su familia asumen cualquier pequeña adversidad postoperatoria, convirtiéndose de este modo en una complicación.

En urología hay un gran número de intervenciones que permiten el desarrollo de este tipo de cirugía, tanto por ser poco invasivas, como por presentar

TABLA I. COMPLICACIONES MAYORES POR AÑOS DE ESTUDIO.

\begin{tabular}{|l|c|c|c|c|}
\hline & $\mathbf{2 0 0 3}$ & $\mathbf{2 0 0 4}$ & $\mathbf{2 0 0 5}$ & $\mathbf{2 0 0 6}$ \\
\hline HEMORRAGIA MAYOR & $1: 504$ & $1: 455$ & $1: 1138$ & $1: 1128$ \\
\hline ANGOR PROLONGADO & $1: 1009$ & 0 & $1: 1138$ & 0 \\
\hline DOLOR TORÁCICO ATIPICO & 0 & $1: 910$ & 0 & 0 \\
\hline INFARTO DE MIOCARDIO & 0 & $1: 910$ & 0 & 0 \\
\hline ACCIDENTE CEREBROVASCULAR & 0 & $1: 910$ & 0 & 0 \\
\hline BRONCOESPASMO & $1: 1009$ & 0 & 0 & 0 \\
\hline BLOQUEO AURICULOVENTRICULAR & 0 & 0 & $1: 1138$ & 0 \\
\hline COMA HIPER-HIPOGLUCÉMICO & 0 & 0 & $1: 1138$ & $1: 1128$ \\
\hline TROMBOBLEBITIS & 0 & $1: 910$ & $1: 1138$ & 0 \\
\hline NEUMONÍA & $1: 1009$ & 0 & 0 & $1: 1128$ \\
\hline SHOCK ANAFILÁCTICO & 0 & $1: 910$ & 0 & 0 \\
\hline PANCREATITIS AGUDA & 0 & 0 & 0 & $1: 1128$ \\
\hline INSUFICIENCIA CARDIACA DESCOMPENSADA & 0 & 0 & $1: 1138$ & $1: 1128$ \\
\hline TAQUIARRITMIAS & $1: 504$ & 0 & 0 & $1: 1128$ \\
\hline COMPLICACIONES TOTALES & $1: 144$ & $1: 130$ & $1: 189$ & $1: 188$ \\
\hline
\end{tabular}


INFECCIÓN HERIDA QUIRÚRGICA

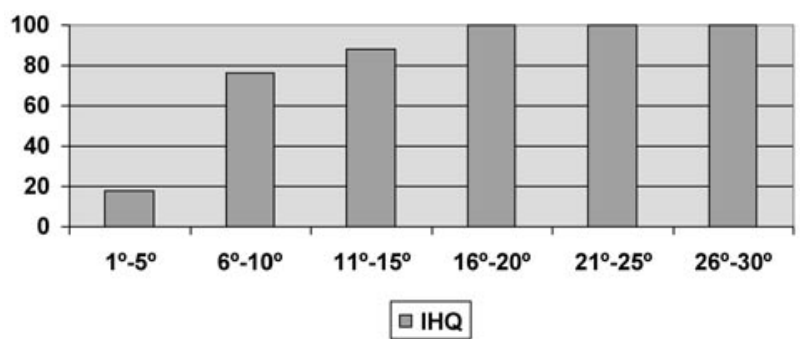

FIGURA 2. Porcentaje de diagnóstico de infección de herida quirúrgica por días tras intervención.

una baja incidencia de complicaciones sistémicas, como por el buen control del dolor mediante analgesia ambulatoria.

En nuestro trabajo el índice de sustitución alcanza la cifra de $83,6 \%$ del total de la actividad del Servicio de Urología. Esta cifra, en parte, es tan elevada debido a que la cirugía de mayor envergadura se desarrolla en un hospital independiente. Además, la lista de intervenciones no es tan amplia como la presentada por Navalón y cols. (6).

El porcentaje de ingresos es del 2,5\%, de los cuales el $85 \%$ fueron ingresos precoces, es decir, pacientes que al finalizar la intervención quirúrgica se reconvierten de forma directa en cirugía de corta estancia, a causa de una serie de factores, entre los que podemos destacar una planificación deficiente en el proceso de selección. De este modo, nuestra tasa de ingresos es menor del $3 \%$, cifra con la que mide la calidad de las unidades de CMA (8), si bien es cierto que nuestros resultados incluyen no sólo la CMA, si no también la cirugía menor ambulatoria.

El índice de suspensión de nuestra serie es de un $12 \%$, muy elevado, respecto a la bibliografía. La presencia de una enfermedad intercurrente es la principal causa de esta elevación del índice de suspensión, por lo que creemos que una opción importante de mejora pudiera ser la realización de una visita o contacto telefónico varios días antes de

TABLA II. COMPLICACIONES MENORES POR AÑOS DE ESTUDIO.

\begin{tabular}{|l|c|c|c|c|}
\hline & $\mathbf{2 0 0 3}$ & $\mathbf{2 0 0 4}$ & $\mathbf{2 0 0 5}$ & $\mathbf{2 0 0 6}$ \\
\hline HEMORRAGIA MENOR & $1: 100$ & $1: 130$ & $1: 126$ & $1: 125$ \\
\hline DOLOR HERIDA QUIRÚRGICA & $1: 84$ & $1: 91$ & $1: 126$ & $1: 112$ \\
\hline CÓLICO RENO-URETRAL & $1: 504$ & $1: 910$ & $1: 284$ & $1: 1128$ \\
\hline SÍNCOPE DE DIVERSA ETIOLOGÍA & 0 & $1: 910$ & 0 & $1: 564$ \\
\hline DOLOR LUMBAR MECÁNICO & 0 & 0 & $1: 1138$ & $1: 564$ \\
\hline RETENCIÓN URINARIA & $1: 504$ & $1: 910$ & 0 & $1: 564$ \\
\hline REACCIÓN ALERGICA & 0 & $1: 910$ & $1: 569$ & 0 \\
\hline CEFALEA POSTPUNCION DURAL & $1: 1009$ & 0 & $1: 1138$ & $1: 1128$ \\
\hline VÉRTIGOS & $1: 504$ & 0 & $1: 1138$ & $1: 1128$ \\
\hline VÓMITOS & 0 & $1: 455$ & $1: 1138$ & $1: 564$ \\
\hline FIEBRE & $1: 336$ & $1: 910$ & $1: 1138$ & $1: 1128$ \\
\hline INFECCIÓN HERIDA QUIRÚRGICA & $1: 252$ & $1: 151$ & $1: 227$ & $1: 376$ \\
\hline ILEO PARALIIICO & $1: 1009$ & $1: 910$ & 0 & 0 \\
\hline CRISIS ANSIEDAD & $1: 1009$ & 0 & 0 & 0 \\
\hline REAGUDIZACION EPOC & 0 & $1: 910$ & $1: 1138$ & $1: 1128$ \\
\hline INFECCIÓN RESPIRATORIA & 0 & $1: 910$ & 0 & 0 \\
\hline INFECCIÓN URINARIA & $1: 504$ & $1: 303$ & $1: 379$ & $1: 564$ \\
\hline COMPLICACIONES TOTALES & & $1: 25$ & $1: 30$ & $1: 30$ \\
\hline
\end{tabular}


la intervención para conocer la presencia de alguna incidencia en el paciente.

Presentamos un total de complicaciones mayores del $0,6 \%$ y menores del $3,6 \%$, teniendo en cuenta que en nuestro programa también incluimos a los pacientes con riesgo ASA III y ASA IV para la cirugía menor ambulatoria. Los estudios afirman que las complicaciones mayores no son frecuentes y la mortalidad es extremadamente baja (9), así, en nuestra serie solo representa un $0,047 \%$, y en los dos casos con una clara relación a la edad y alta comorbilidad asociada del paciente, sin relación con la técnica anestésico-quirúrgica empleada. Los casos de infarto de miocardio, embolias pulmonares o déficit cerebrales son extremadamente bajos, con una incidencia tan baja como la que puede esperarse por la edad del paciente en grupos sin intervención quirúrgica (10). Entre las complicaciones menores predomina el dolor postoperatorio, que por otro lado puede ser un factor desencadenante de vómitos, dehiscencias de sutura con el consiguiente riesgo de hemorragia (11). Se debe potenciar en este tipo de unidades los protocolos de analgesia domiciliaria en todo tipo de intervenciones desarrolladas. La formación a los distintos especialistas quirúrgicos de la importancia del tratamiento del dolor y las distintas formas de analgesia postoperatoria, así como la importancia de la medicación de rescate es un punto de la Unidad que debe ser mejorado en un futuro cercano. El dolor moderado-severo es posible tratarlo con seguridad y de modo eficaz en el domicilio del paciente.

La vigilancia de la infección quirúrgica de modo tradicional debido a la disminución de los días de hospitalización tiende a quedar obsoleta, y su seguimiento tras el alta hospitalaria adquiere cada vez mayor importancia. Varios investigadores $(12,13)$, han demostrado que la mayoría de estas infecciones se evidencian a las 3 semanas postintervención, estos datos son similares con nuestros hallazgos, pues en nuestra serie a los 16 días se detectan la inmensa mayoría de las infecciones quirúrgicas de nuestro medio.

Es destacable el porcentaje de pacientes, ya mencionado, que, mediante encuesta telefónica, han mostrado su satisfacción por haber participado en éste tipo de cirugía.

No comparamos nuestros resultados con el de otros trabajos publicados previamente por dos razones, en primer lugar porque no todos los programas de cirugía ambulatoria asumen las mismas intervenciones, y en segundo lugar porque en nuestro trabajo, los resultados ofrecidos incluyen el total de la cirugía ambulatoria, es decir, tanto la cirugía menor como la CMA.
En conclusión, podemos afirmar que el auge y la promoción continua de la cirugía ambulatoria está justificada en estas patologías que no asocian una gran complejidad, tras observar la satisfacción de los pacientes sometidos a este tipo de cirugía, como tras analizar el escaso número de complicaciones registradas, conllevando un importante desahogo asistencial y un aumento de la efectividad de las diferentes unidades quirúrgicas.

\section{BIBLIOGRAFIA y LECTURAS RECOMENDADAS ( ${ }^{*}$ lectura de interés $y^{* *}$ lectura fundamental)}

*1. VALERO, J.; MEDINA, M.; GUERRERO, J. y cols.: "Realización de cirugía mayor ambulatoria dentro de una unidad integrada". Actas Urol. Esp., 23: 523, 1999.

**2. VARGAS, C.; RIUS, G.: "Cirugía urológica sin ingreso". Actas Urol. Esp., 17: 87, 1993.

*3. SAMPIETRO, A.; VAQUERIZO, A.; FERNANDEZ, A. y cols.: "Cirugía mayor ambulatoria en Urología: Nuestra experiencia”. Arch. Esp. Urol., 48: 343, 1995.

*4. LLOPIS, B.; NAVARRO, J.A.; MOLA, M.J. y cols.: "Cirugía mayor ambulatoria en urología. 5 años de experiencia". Actas Urol. Esp., 27: 117, 2003.

**5. DAVIS, J.E.: "Major Ambulatory Surgery". Baltimore, Williams \& Wilkins 1986.

*6. NAVALON, P.; ZARAGOZA, C.; CANOVAS, J.A. y cols.: "Cirugía Mayor Ambulatoria en Urología. Análisis de nuestra experiencia". Arch. Esp. Urol., 57: 513, 2004.

*7. MARTÍN MORALES, J.: "Cirugía mayor ambulatoria: Una transformación necesaria”. Cirugía Mayor Ambulatoria, 1: 133, 1996.

*8. JIMENEZ CALVO, J.; RAIGOSO, O.; PINOS, M. y cols.: "Cirugía mayor ambulatoria: Nuestra experiencia". Actas Urol. Esp., 26: 384, 2002.

*9. VILA, M.: "Reflexiones sobre la morbimortalidad ligada a la anestesia en cirugía mayor ambulatoria". Cir. May. Amb., 1: 44, 1996.

10. SCOTT, I.; CHUNG, F.: "Discharge criteria and complications alter ambulatory surgery". Anesth. Analg., 88: 508, 1999.

11. CHUNG, F.; RITCHIE, E.; SU, J.: "Postoperative pain in ambulatory surgery". Anesth. Analg., 85: 808, 1997.

12. WEIGELTIT, A.; DRYER, D.; HALEY, R.W.: "The necessity and efficency of wound surveillance after discharge". Arch. Surg., 127: 77, 1992.

13. MERTENS, R.; JANS, B.; KURZ, X.: "A computerized nationwide network: For nosocomial infection surveillance in Belgium". Infect Control Hosp. Epidemiol., 15: 171, 1994. 